La question des conditions aux limites d'un écoulement est récurrente en mécanique des fluides. L'hypothèse usuelle de non-glissement d'un liquide sur une paroi solide est vérifiée à l'échelle macroscopique, mais pas toujours à plus petite échelle. Au-delà de l'intérêt fondamental de la compréhension

de la friction interfaciale, le glissement d'un liquide sur un solide est devenu un enjeu pratique important pour les écoulements confinés.

Nous avons conçu un appareil de mesure dynamique des forces de surfaces, qui permet de déterminer, de façon fiable et précise, les conditions aux limites de l'écoulement d'un fluide. Nous avons ainsi pu démontrer I'influence cruciale des propriétés de surface sur le glissement d'un liquide simple sur une paroi.

\title{
Propriétés hydrodynamiques au voisinage d'une surface
}

\author{
Élisabeth Charlaix ${ }^{(1)}$, Cécile Cottin-Bizonne ${ }^{(1)}$, Jérôme Crassous ${ }^{(2)}$, Samuel Leroy ${ }^{(1)}$, \\ Frédéric Restagno $^{(3)}$ (restagno@|ps.u-psud.fr), Audrey Steinberger ${ }^{(4)}$ \\ (1) Laboratoire de physique de la matière condensée et nanostructures, Université Claude Bernard Lyon 1 et CNRS, \\ 43, bd du 11-Novembre 1918, 69622 Villeurbanne Cedex \\ (2) Institut de physique de Rennes, UMR CNRS 6251, Université de Rennes 1, 35042 Rennes Cedex \\ (3) CNRS, Laboratoire de physique des solides, Bât. 510, Campus universitaire d'Orsay, 91405 Orsay Cedex \\ (4) Laboratoire de physique, CNRS - ENS Lyon, 69364 Lyon Cedex 07
}

\section{Position du problème}

Si la mécanique des fluides est une science ancienne, puisqu'une bonne part des grands noms qui lui sont attachés remontent aux $18^{\mathrm{e}}$ et $19^{\mathrm{e}}$ siècles (Euler, Bernoulli, Navier, Stokes...), ses applications sont nombreuses et vont de la compréhension de l'éjection de l'ADN hors de la coque protéique des virus, à la description de la dynamique des nébuleuses célestes. Dans le cas des fluides visqueux les plus simples, dont la viscosité ne dépend pas du taux de cisaillement ${ }^{(1)}$ et qui sont appelés fluides newtoniens, l'évolution de la vitesse à l'intérieur du fluide est décrite par l'équation de Navier-Stokes. Cette équation non linéaire aux dérivées partielles est célèbre au-delà du monde des physiciens, car elle fait partie des "sept problèmes du prix du millénaire " dont la résolution conduira à l'attribution d'un chèque d'un million de dollars.

Pour résoudre cette équation, il faut en connaître les conditions aux limites, c'està-dire la valeur des champs de vitesse et de contrainte à la frontière du domaine qui limite le fluide. Dans le cas d'un fluide s'écoulant au voisinage d'une paroi solide immobile, la non-pénétration du fluide dans le solide impose que la composante de vitesse perpendiculaire à la surface de séparation du fluide et du solide soit nulle. Il n'y a pas d'argument aussi simple pour définir la valeur de la composante de vitesse parallèle à la paroi. La réponse universellement enseignée dans les livres est celle que R. Feynman énonce dans son cours de physique de la façon suivante : «Il se trouve - bien que ce ne soit absolument pas évident en soi - que, dans toutes les conditions où l'on a pu le vérifier expérimentalement, la vitesse d'un fluide est rigoureusement nulle à la paroi solide » [1].
En fait, grâce aux progrès des moyens d'investigation à petite échelle depuis une vingtaine d'années, cette hypothèse a été remise en question, alors qu'il s'agissait d'un dogme bien établi dans le cas des liquides simples. De nombreuses méthodes expérimentales ont été développées pour relever le défi que constitue ce problème de la détermination du glissement - ou non - d'un liquide sur un solide, et une partie des résultats semble contredire l'hypothèse du non-glissement [2].

\section{La longueur de Navier}

Dans le cas où l'on imagine qu'il y a glissement, il est possible de le caractériser par plusieurs grandeurs, comme représenté sur la figure 1. La vitesse de glissement $v_{\mathrm{g}}$ représente la vitesse tangentielle du fluide à la paroi solide, tandis que la longueur de glissement $b$ - aussi appelée longueur de Navier - représente la profondeur, à l'intérieur du solide, pour laquelle la vitesse s'annulerait en supposant un profil de vitesse linéaire.

On montre, à partir de l'équation de Navier-Stokes, que le fluide (de viscosité $\eta$ ) qui s'écoule sur le solide lui transmet une force par unité de surface proportionnelle au gradient de vitesse $: \eta \mathrm{d} v / \mathrm{d} z=\eta v_{\mathrm{g}} / b=k v_{\mathrm{g}}$, c'est-à-dire une force de friction simplement proportionnelle à la vitesse de glissement et parallèle à la paroi. Suivant la technique expérimentale utilisée, on mesurera en fait soit la vitesse de glissement, soit la longueur de glissement. Il est alors intéressant de tester l'indépendance de cette longueur par rapport au taux de cisaillement, car cela permet de voir s'il existe une relation linéaire entre force et vitesse - et donc un coefficient de friction $k$ intrinsèque $-c e$ qui n'est pas évident a priori. 


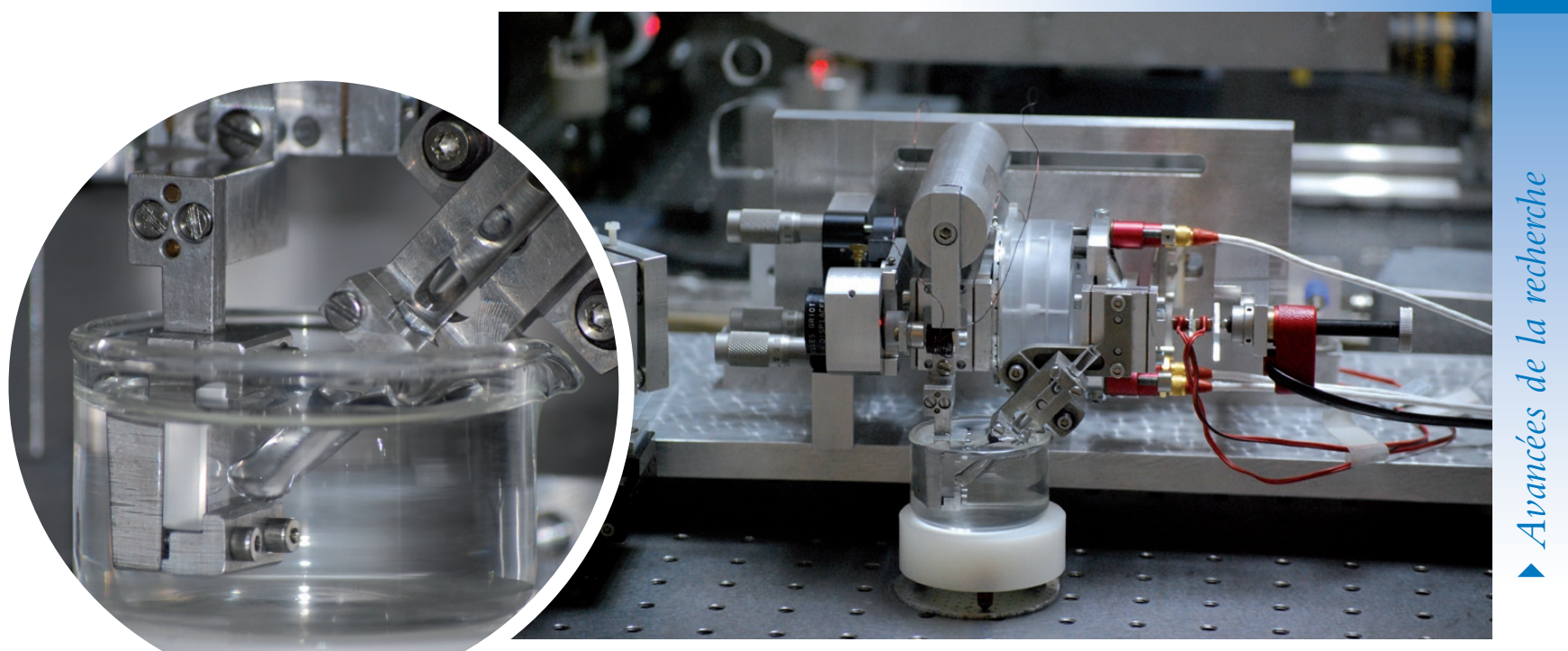

Dispositif de mesure des forces hydrodynamiques entre un plan et une sphère, dont le schéma est représenté sur la figure 2, p. 16. @ S. Leroy / LPMCN (CNRS et Université Claude-Bernard Lyon 1).
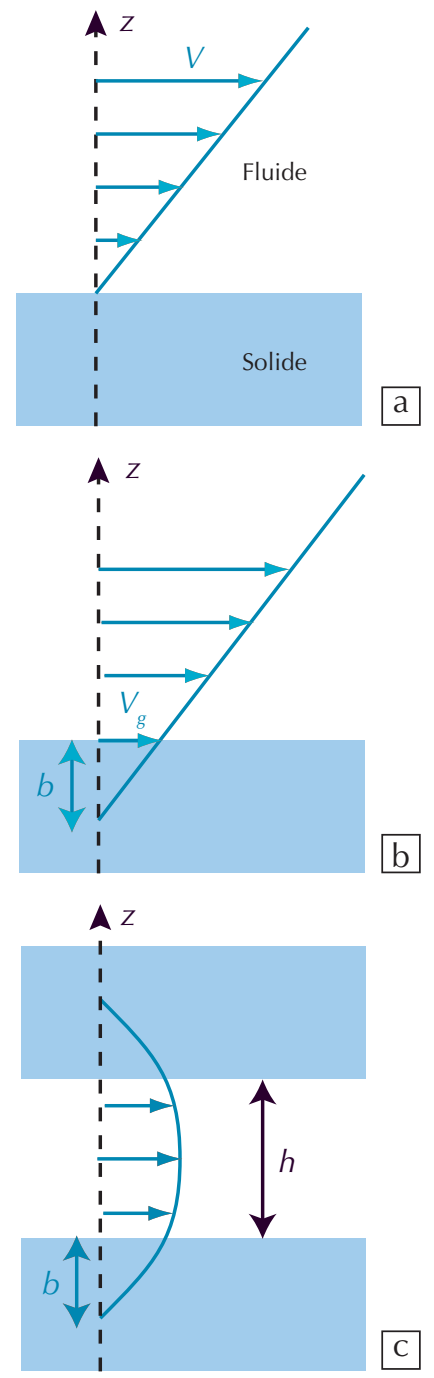

1. Conditions de vitesse d'un fluide (indiquée par les flèches bleues) à une interface fluide-solide.

(a) Écoulement classique d'un fluide visqueux en l'absence de glissement.

(b) Écoulement d'un fluide en présence d'un glissement : la vitesse du liquide à la paroi, $v_{g}$, est non nulle. La vitesse s'annulerait à l'intérieur du solide, à une profondeur $b$. (c) Écoulement d'un fluide dans une veine liquide de largeur $h$. Tout se passe comme si la veine liquide avait une largeur $h+2 b$.
Des conséquences potentiellement importantes

En fait, si l'on considère un écoulement à l'intérieur d'une veine liquide d'épaisseur $h$, comme représenté sur la figure $1 c$, l'existence de la longueur de glissement conduit à considérer que le tube a une taille effective plus grande, de l'ordre de $h+2 b$. Les longueurs de glissement étant petites (sinon on n'aurait pas autant de mal à les mesurer) et indépendantes de la taille du canal, l'impact de la présence de glissement sur les écoulements sera donc d'autant plus important que le confinement $h$ est de taille comparable à $b$.

La course à la miniaturisation, motivée par le développement des microsystèmes pour l'analyse chimique, des laboratoires sur puces, des applications des matériaux nanoporeux ou autres nanofluides, requiert une description fine des phénomènes de transport à l'interface entre le liquide et le solide. L'enjeu est non seulement d'assurer un bon contrôle des écoulements dans de tels systèmes, mais aussi d'améliorer leur efficacité en utilisant le glissement pour diminuer les pertes de charge $^{(2)}$ dans les micro- et nanocanaux. À un niveau plus fondamental, il s'agit de comprendre l'origine physique et les mécanismes de la friction à l'interface.

\section{Des réponses expérimentales variées}

\section{Le cas particulier des polymères}

Dans le cas des fondus de polymères, l'existence d'une longueur de glissement $b$ pouvant atteindre quelques micromètres ne fait pas de doute. La grande valeur de $b$ a alors permis de lever toute ambiguïté expérimentale : Mooney, en 1931, fut le premier à mesurer une augmentation du débit d'un polymère fondu à travers un capillaire par rapport à la valeur attendue en l'absence de glissement, et ce résultat a été clairement confirmé par des expériences ultérieures de mesure de la vitesse près d'une paroi solide [3].

\section{Les fluides simples}

Le cas des fluides simples ou des petites molécules est beaucoup plus complexe. Les résultats obtenus ces dix dernières années par différentes techniques de mesure présentent une dispersion assez large. La diversité est importante, non seulement au niveau de l'amplitude des effets de glissement observés, qui different de plusieurs ordres de grandeur, mais également qualitativement, sur l'écart à la linéarité de la relation force-vitesse et sur l'influence du taux de cisaillement sur la valeur de $b$. Pour ne citer que le cas de l'eau ou de mélanges de solutions aqueuses (glycérol, sucrose), il a été reporté sur des surfaces lisses des longueurs de glissement allant de quelques nanomètres à quelques micromètres. Cette grande variabilité des résultats expérimentaux n'est pas nouvelle : le débat avait déjà fait rage au temps de Stokes, même si les résolutions expérimentales d'alors étaient différentes. En effet, mesurer des écoulements aux interfaces constitue un véritable défi expérimental.

Il existe deux types de méthodes de mesure.

- Les méthodes dites directes, qui cherchent à déterminer les vitesses au voisinage des surfaces, mesurent en général la vitesse de traceurs ensemençant le fluide. Une façon possible de ne suivre que les traceurs proches de la surface est de les illuminer grâce à une onde lumineuse évanescente. 
>>

Si ces traceurs sont des particules, ils sont sujets aux interactions colloïdales et au mouvement brownien au voisinage de la surface, dont la correction demande beaucoup d'attention.

- Une autre catégorie de méthodes, les méthodes de dissipation, visent à caractériser la friction à l'interface en mesurant les corrections à la perte de charge qui serait obtenue sans glissement. Pour ce type d'expériences, il est crucial de contrôler très précisément la valeur de la viscosité du liquide étudié, ainsi que la géométrie du système. Par exemple, dans le cas d'un écoulement dans un capillaire cylindrique de rayon $R$, on mesure le rapport débit/pression qui varie en $R^{4}$, tandis que les effets de glissement à la surface apportent une correction en $b / R$. Les méthodes de dissipation deviennent d'autant plus sensibles que le confinement du liquide est important. Cette propriété est mise à profit dans les techniques utilisant un appareil de mesure dynamique des forces de surface, tel que celui présenté dans cet article.

Enfin, un dénominateur commun à toutes les méthodes est l'importance du contrôle de la surface elle-même : pour caractériser un glissement "intrinsèque » il est nécessaire d'avoir une surface lisse, sans poussière et homogène d'un point de vue chimique.

\section{Appareil de mesure dynamique des forces de surfaces}

Afin d'obtenir une détermination fiable et précise des conditions aux limites hydrodynamiques, nous avons construit un appareil dédié aux mesures dynamiques de nanorhéologie [4] (fig. 2). Cet appareil permet de mesurer la force qui s'exerce entre deux surfaces (en l'occurrence une sphère de rayon centimétrique et un plan) entre lesquelles est introduit le liquide, tout en contrôlant la distance $h$ entre celles-ci, à une fraction de nanomètre près. En mode dynamique, le système est excité par une vibration de petite amplitude (typiquement $1 \mathrm{~nm}$ ) et de pulsation $\omega$ de la sphère dans la direction normale au plan, appliquée au moyen d'une céramique piézo-électrique. Le plan est monté sur un ressort de raideur connue (en fait un bilame élastique pour assurer un bon guidage), dont la déformation est mesurée grâce à un capteur optique. La force exercée sur le plan est alors simplement égale à la déformation du bilame multipliée par sa raideur. Le déplacement relatif entre

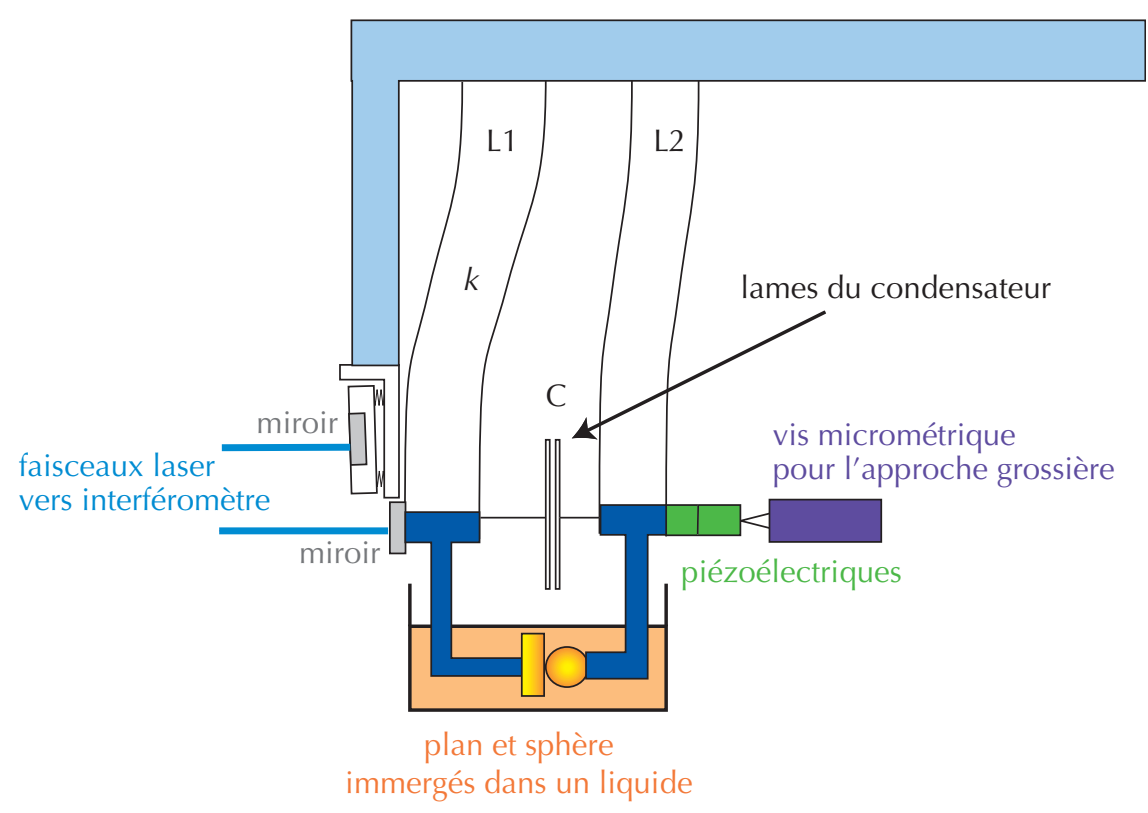

2. Dispositif de mesures nanorhéologiques. On utilise un système macroscopique : un plan et une sphère de dimensions centimétriques, dont les déplacements sont contrôlés à l'échelle de l'ångström. Les deux surfaces sont montées sur des bilames élastiques (L1 et L2) pour un guidage linéaire à un degré de liberté. La sphère est mise en mouvement par des éléments piézoélectriques et le déplacement du plan donne accès à la force d'interaction. Ce déplacement est mesuré au voisinage du point de force nulle, par un interféromètre de type Nomarsky de très grande résolution. Le déplacement relatif des deux surfaces est mesuré par un capteur capacitif $\boldsymbol{C}$, de résolution de l'ordre de l'ångström sur une gamme de plusieurs micromètres. Ce dispositif permet des mesures de la réponse dynamique dans une gamme de fréquences allant de 5 à $100 \mathrm{~Hz}$.

la sphère et le plan est, quant à lui, mesuré par un capteur capacitif. La résolution statique est de $0,15 \mu \mathrm{N}$ sur les forces et de $0,05 \mathrm{~nm}$ sur le déplacement ; les résolutions dynamiques correspondantes sont de $8 \mathrm{nN}$ et de $0,002 \mathrm{~nm}$.

Dans les expériences de nanorhéologie, les surfaces sont immergées dans un liquide. Le mouvement oscillant de la sphère impose un écoulement de drainage entre les surfaces. L'appareil mesure la force visqueuse $F$ induite par la surpression liée à cet écoulement, et qui est proportionnelle à la vitesse de la sphère ${ }^{(3)} ; F$ est donc, comme la vitesse, en quadrature de phase avec le déplacement : la fonction de réponse dynamique $G(\omega)$ (rapport de la force au déplacement) est donc imaginaire en notation complexe.

En l'absence de glissement, cette partie imaginaire G' $(\omega)$ caractérisant la dissipation visqueuse vérifie la formule de Reynolds :

$$
G^{\prime \prime}(\omega)=6 \pi \eta R^{2} \omega / h \quad \text { (1) }
$$

où $\eta$ est la viscosité du liquide et $R$ est le rayon de la sphère. Son inverse $G^{\prime \prime-1}(\omega)$ varie donc linéairement avec la distance moyenne entre les surfaces, $h$ (comme la droite en noir de la figure 3).

Dans le cas d'un glissement partiel sur le plan, cette formule doit être corrigée par un facteur $f^{\star}$ qui ne dépend que du rapport entre la longueur de glissement $b$ et la distance $h$ :

$$
G^{\prime \prime}(\omega)=\left[6 \pi \eta R^{2} \omega / h\right] f^{\star}(b / h)
$$

On trouve alors analytiquement que la courbe de variation de l'inverse de l'amortissement visqueux n'est plus une simple droite mais une courbe qui tend vers zéro à petite distance $\left(\lim _{h \rightarrow 0} G^{\prime \prime}-1(\omega)=0\right)$, et qui tend asymptotiquement à grande distance $(h>>b)$ vers une droite dont la position dépend de $b$ (courbe bleue de la figure 3).

En pratique, l'origine des distances, $h=0$, est simplement déterminée par la position pour laquelle la dissipation diverge (et donc $G^{\prime-1}(\omega)$ tend vers zéro). La longueur de glissement est déterminée indépendamment de la viscosité du liquide ou du rayon de la sphère, si l'on peut mesurer $G(\omega)$ dans une large gamme de distances. C'est le cas de notre dispositif, qui présente une excellente résolution en régime dynamique.

\section{Glissement de l'eau}

\section{Les surfaces lisses}

Dans un premier temps, le glissement a été mesuré sur des surfaces très lisses [5]. 


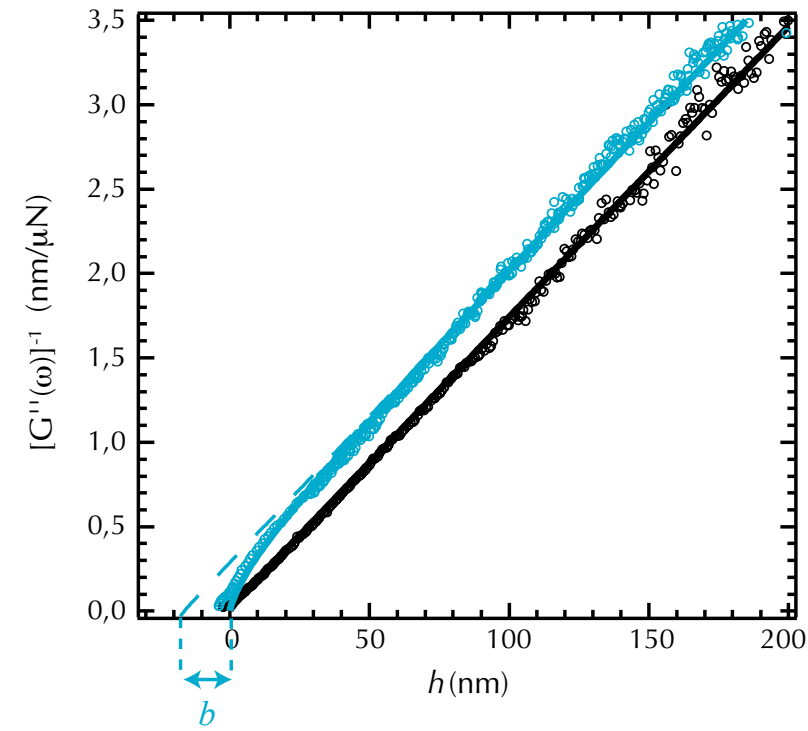

3. Glissement et non-glissement de l'eau sur des surfaces lisses mouillantes et non mouillantes. Inverse de l'amortissement visqueux mesuré avec de l'eau confinée entre une sphère et un plan. Entre des surfaces lisses hydrophiles (pyrex flotté de rugosité moyenne quadratique $4 \AA$, en noir), l'eau se comporte comme un fluide newtonien sans glissement sur les surfaces : $G^{\prime \prime-1}(\omega)$ varie proportionnellement à la distance $h$ entre le plan et la sphère. Sur un plan hydrophobe (pyrex silanisé, en bleu) apparaît un glissement correspondant à une longueur de Navier de $17 \pm 2 \mathrm{~nm}$, constante sur toute la plage de mesure. Les lignes pleines correspondent au meilleur ajustement avec l'expression (2) du texte. La position du zéro est obtenue sans paramètre ajustable.

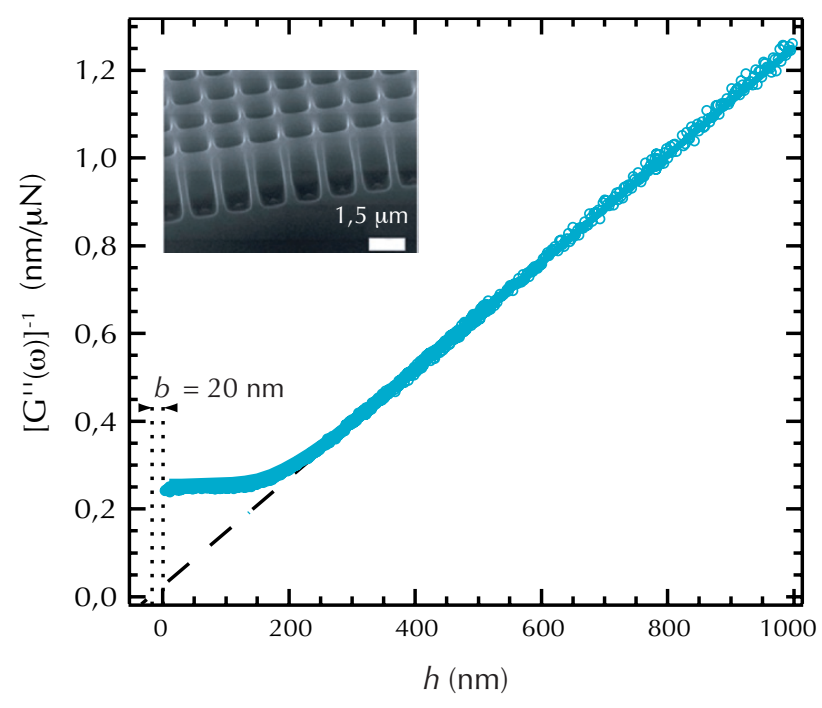

4. Glissement de l'eau sur un tapis de bulles. L'inverse de l'amortissement visqueux varie proportionnellement à la distance $h$ entre la sphère et le plan à grande distance, faisant apparaitre une longueur de glissement de $20 \mathrm{~nm}$. La position du zéro est obtenue quand la sphère vient au contact du plan. À courte distance, la dissipation visqueuse sature.

Insert : image en microscopie électronique des surfaces superhydrophobes utilisées.
Nous avons utilisé du pyrex flotté : sa surface a une rugosité quadratique moyenne de $0,4 \mathrm{~nm}$ (mesurée sur $4 \mu \mathrm{m}^{2}$ par microscopie à force atomique) et est facilement fonctionnalisable. Nous comparons ainsi un plan de pyrex nu hydrophile et un plan de pyrex rendu hydrophobe par greffage de chaînes hydrocarbonées ${ }^{(4)}$, sur lequel l'eau a un angle de contact de 110 degrés.

On voit nettement sur la figure 3 que l'écoulement de l'eau sur le pyrex hydrophile vérifie une condition limite de non-glissement : en effet, la variation de $G$ "-1 $(\omega)$ avec la distance $h$ est représentée par une droite qui passe par l'origine (déterminée par la position pour laquelle les surfaces solides entrent en contact), ce qui est en bon accord avec la formule de Reynolds (eq. 1). La résolution est ici uniquement limitée par l'incertitude sur la position du contact rigide entre les surfaces et vaut $3 \mathrm{~nm}$.

En revanche, un glissement net est observé sur la surface rendue hydrophobe. L'ajustement avec l'expression (2), en utilisant l'expression analytique de la fonction $f^{\star}$, permet de déterminer une longueur de glissement $b=17 \pm 2 \mathrm{~nm}$. Cette longueur de glissement est l'unique paramètre ajustable de la théorie, qui est en parfait accord avec les données expérimentales. Par ailleurs, $b$ est indépendant du taux de cisaillement dans la gamme des taux de cisaillement utilisés (jusqu'à $510^{3} \mathrm{~s}^{-1}$ ), ce que l'on vérifie en modifiant l'amplitude et la fréquence de l'excitation imposée. Cette caractérisation expérimentale de la condition limite implique donc une relation force-vitesse à la paroi strictement linéaire dans toute la plage de paramètres que nous avons explorée.

\section{Les surfaces rugueuses}

En fait, les expériences sur les fluides simples comme l'eau sont extrêmement sensibles à la rugosité des surfaces. De plus, dans le cas des surfaces hydrophobes, la rugosité peut conduire à un état dit de "superhydrophobicité ", c'est-à-dire au piégeage de poches d'air entre le liquide et le solide. La question est alors la suivante : la rugosité va-t-elle freiner le mouvement relatif ou au contraire le faciliter, si des poches d'air peu visqueux apparaissent entre la surface et le liquide [6] ?

Nous avons étudié l'écoulement du glycérol, un liquide polaire et donc de grande tension de surface, entre une sphère de pyrex identique à celles utilisées dans les expériences précédentes et une surface rugueuse superhydrophobe [7] (insert de la fig. 4). Cette surface est celle d'une pastille de silicium gravée de trous cylindriques de rayon $0,65 \mu \mathrm{m}$ et de profondeur 3,5 $\mu \mathrm{m}$, disposés sur un réseau carré de pas $1,4 \mu \mathrm{m}$. Elle a été rendue hydrophobe à l'aide du même traitement chimique que dans le cas des surfaces lisses discuté cidessus : comme on évite ainsi le remplissage des trous par le glycérol, on pourrait s'attendre à observer un très grand glissement dû à la lubrification par l'air. En fait, les résultats sont plus surprenants.

Tout d'abord, il est remarquable que la courbe expérimentale (fig. 4) ne suive pas l'équation (2), car un plateau apparaît quand les surfaces sont presque au contact. Ce plateau est en fait dû à l'élasticité des bulles piégées dans les trous. Près du contact, la déformation des bulles en réponse à la variation de pression associée à l'écoulement compense le déplacement de la sphère, sans qu'il soit nécessaire d'éjecter du liquide vers l'extérieur : le liquide pénètre dans les trous, de telle sorte que l'épaisseur du film liquide entre les surfaces et aussi la dissipation restent constantes. Toutefois la déformation des bulles a une influence négligeable sur la 
dissipation à grande distance $h$, et donc n'affecte pas la mesure de la longueur de glissement effective $b$. Dans ce cas où l'équation (2) ne s'applique plus à faible distance, il faut déterminer la position de l'origine des distances différemment : elle est alors définie par la position du point de contact entre les surfaces, avec une incertitude de $\pm 10 \mathrm{~nm}$ dans ces expériences. On trouve alors pour le glycérol sur ces surfaces une longueur de glissement de $20 \pm 10 \mathrm{~nm}$.

Pourquoi un glissement aussi faible par rapport aux attentes? En fait, il est facile de se convaincre qu'il n'y a aucune raison que les bulles remplissent les trous de la structure superhydrophobe juste à ras bord. La présence d'un sur-remplissage ou d'un sous-remplissage modifie, comme la rugosité, la forme des lignes de champ de l'écoulement et donc le transfert de quantité de mouvement à l'interface.

Pour mieux comprendre cet effet, nous avons réalisé des simulations numériques par éléments finis du glissement d'un liquide sur un tapis de bulles plus ou moins bombées [8]. Dans ces simulations, le liquide glisse parfaitement sur la surface des bulles, modélisées par des calottes sphériques accrochées sur les arêtes des trous de la surface, et ne glisse pas sur la paroi solide entre les bulles. Le résultat est représenté sur la figure 5. La longueur de glissement théorique est une fonction non monotone de l'angle de remplissage $\theta$.
Comme on peut s'y attendre, cette longueur de glissement est maximale dans le cas où la surface des bulles ne crée ni bosses ni creux $(\theta=0)$; puis, elle décroît quand l'angle de remplissage augmente. On peut même trouver des situations où elle devient négative quand les bulles sont très remplies. Ceci n'a rien de paradoxal, et correspond juste au fait qu'une grande rugosité déplace aussi la position moyenne de l'interface vers l'intérieur du liquide. La valeur de la longueur de glissement mesurée dans les expériences ( $20 \mathrm{~nm}$, fig. 4) correspond alors à des bulles bombées vers l'extérieur, avec un angle $\theta$ de $45^{\circ}$ (pour cette expérience).

\section{Conclusion}

En conclusion, nous avons reconsidéré dans ce travail, grâce à un appareil original, un vieux dogme de la mécanique des fluides classique : celui de la condition aux limites à la paroi. Si le glissement des polymères était bien connu, le comportement des fluides simples est plus complexe. Dans le cas d'un écoulement d'eau sur des surfaces lisses, nous avons montré qu'il peut exister un glissement d'autant plus important que ces surfaces sont hydrophobes. La longueur de glissement est très sensible à de petits changements de détails de la surface : nanorugosité, composition chimique. Il est donc envisageable d'obtenir dans certains cas des longueurs de glissement encore plus importantes, en optimisant les propriétés de surface. Cependant, la voie des surfaces topographiquement texturées est plus décevante qu'on aurait pu le penser naïvement, et des calculs ont également montré qu'une toute petite fraction de contact solide-liquide suffit à annuler l'effet de la lubrification par une grande fraction de poches d'air. La mise en mouvement d'un liquide dans des nanocanaux conduit donc toujours, pour l'instant, à des pertes de charge très importantes.

Pour conclure de façon plus optimiste, même un glissement modéré pourrait être suffisant pour contribuer à des effets importants dans le cas d'écoulements générés par des phénomènes interfaciaux, par exemple par « électro-osmose » (mise en mouvement d'un liquide par l'application d'un champ électrique en présence de charges de surfaces), ou encore par " diffusio-phorèse ", qui prédit la mise en mouvement d'un liquide par un gradient de concentration au voisinage des surfaces.

(1) "Taux de cisaillement " est une autre appellation de "gradient de vitesse ". Celui-ci se mesure en $\mathrm{s}^{-1}$. C'est une mesure de la non-uniformité de la quantité de mouvement entre différents endroits du fluide.

(2) La perte de charge est la différence de pression entre deux points d'une canalisation, qui est nécessaire pour mettre un liquide en écoulement en luttant contre les forces de viscosité.

(3) La force visqueuse mesurée est perpendiculaire au plan, car la friction modifie la forme de l'écoulement, et donc le profil de pression dans le liquide.

(4) Une monocouche d'octadécyl trichlorosilane.

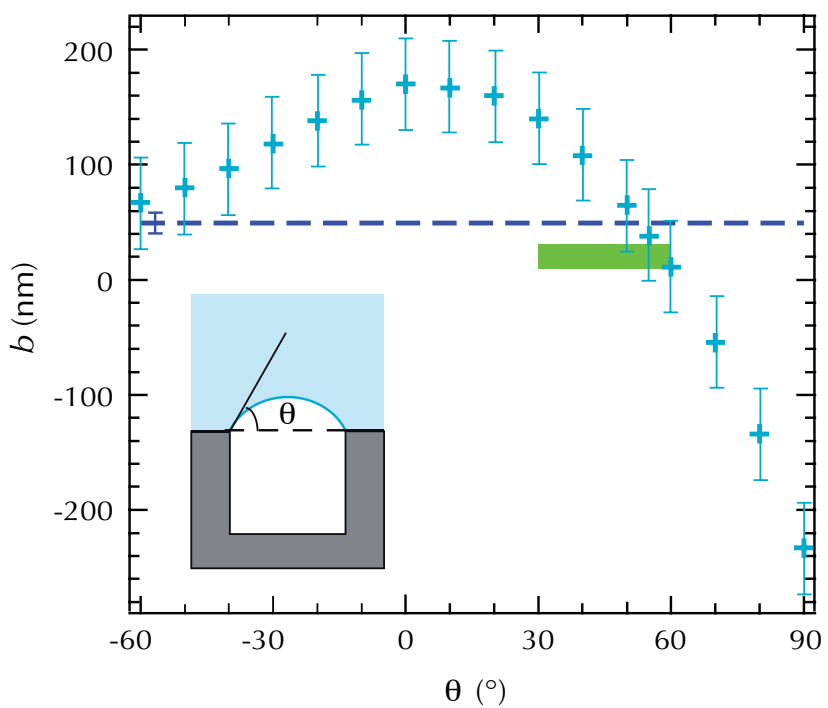

5. Évolution du glissement de l'eau sur un tapis de bulles en fonction de l'angle de remplissage, $\theta$. Les croix bleues correspondent à une prédiction théorique, la ligne en tirets bleu foncé correspond à la prédiction théorique lorsque le liquide envahit les trous. Le rectangle vert correspond à la valeur mesurée ( $b=20 \pm 10 \mathrm{~nm}$ ).

\section{Références}

1 - "Le cours de physique de Feynman Électromagnétisme 2 ", Dunod (1999). Les deux chapitres "L'écoulement de l'eau sèche » et « L'écoulement de l'eau mouillante » sont une bonne introduction à la mécanique des fluides.

2 - Pour une revue : C. Nieto et al., "Boundary slip in Newtonian liquid: a review of experimental studies", Rep. Prog. Phys. 68 (2005) 2859-2897.

3 - K.B. Migler et al., Phys. Rev. Lett. 70 (1993), 287-291.

4 - F. Restagno et al., Rev. Sci. Instrum. 73 (2002), 2292-98.

5 - C. Cottin-Bizonne et al., Phys. Rev. Lett. 94 (2005) 056102

6 - M. Reyssat et D. Quéré, "L'effet lotus », Pour la Science, n³47 (septembre 2006).

7 • A. Steinberger et al., Phys. Rev. Lett. 100 (2008) 134501.

8 - A. Steinberger et al., Nature Materials 6 (2007), 665-668. 